\title{
226 Myocardial fibrosis, wall thickness, and microvascular perfusion in hypertrophic cardiomyopathy. a cardiovascular magnetic resonance study
}

\author{
Rory O'Hanlon*1, Agata Grasso ${ }^{1}$, Chiara Bucciarelli-Ducci ${ }^{1}$, Rick Wage ${ }^{1}$, \\ Meghana Kulkarni², Ali Alsafi², Peter Gatehouse1, Roughton Micheal², \\ Dudley J Pennell ${ }^{1}$ and Sanjay K Prasad ${ }^{1}$
}

Address: ${ }^{1}$ Royal Brompton Unit, London, UK and ${ }^{2}$ Imperial College, London, UK

* Corresponding author

from I th Annual SCMR Scientific Sessions

Los Angeles, CA, USA. I-3 February 2008

Published: 22 October 2008

Journal of Cardiovascular Magnetic Resonance 2008, I0(Suppl I):A87 doi:I0.I I86/I532-429X-10-SI-A87

This abstract is available from: http://jcmr-online.com/content/I0/SI/A87

(c) 2008 O'Hanlon et al; licensee BioMed Central Ltd.

\begin{abstract}
Introduction
HCM is the commonest cause of sudden cardiac death in young adults and an important cause of heart failure. An important trigger for malignant arrhythmic events in this cohort is the presence of myocardial fibrosis and myocardial ischaemia due to abnormal microvascular function and/or a mismatch between areas of increased LV mass and coronary flow. Current risk stratification algorithms do not include degrees of fibrosis or severity of microvascular dysfunction, but these parameters may account for increased risk in those with greatest degrees of hypertrophy.
\end{abstract}

\section{Purpose}

Myocardial fibrosis and microvascular ischaemia are common findings in hypertrophic cardiomyopathy (HCM). We sought to assess the correlation between wall thickness, severity of perfusion abnormalities, and degrees of myocardial fibrosis in patients with HCM.

\section{Methods}

We prospectively scanned 50 patients with HCM using the same protocol on a $1.5 \mathrm{~T}$ Siemens Avanto scanner. Following standard long and short axis cine imaging, adenosine $140 \mathrm{mcg} / \mathrm{kg} / \mathrm{min}$ was infused for 4 minutes and first-pass perfusion imaging was then performed using a 3 slice hybrid-EPI sequence (TR $5.8 \mathrm{~ms}, 30^{\circ}$, ETL 4, $1860 \mathrm{~Hz} /$ pixel, $2.8 \times 2.8 \times 8 \mathrm{~mm}$ voxels over typically $360 \times 270$ mm FOV (adapted per patient) at TI $=110-140 \mathrm{~ms}$ after BIR-4 saturation for each of the 3 fat-suppressed slices per cycle, using TSENSE (R2, coil profiles average 8)). Gadolinium contrast dose used for $1^{\text {st }}$ pass was $0.1 \mathrm{mmol} / \mathrm{kg}$ at followed by a $15 \mathrm{~mL}$ saline flush at $7 \mathrm{~mL} / \mathrm{s}$. Following stress perfusion, a series of short axis LGE images were acquired from base to apex to quantify fibrosis using an inversion-recovery segmented-FLASH imaging sequence (TR $6.6 \mathrm{~ms}, 25^{\circ}, 23$ lines per cycle, $230 \mathrm{~Hz} /$ pixel, $1.4 \times 2.3$ $\times 8 \mathrm{~mm}$ voxels over typically $360 \times 270 \mathrm{~mm}$ FOV (adapted per patient) with TI adjusted to null normal myocardium, and alternate cycle triggering)). Two operators blinded to patient clinical details analyzed the scans to determine peak end-diastolic wall thickness on a per segment basis using the AHA/ACC 17 segment model. The percentage transmurality of perfusion abnormalities and fibrosis was quantified visually according to the following scoring system: $0=$ none, $1=1-24 \% ; 2=25-49 \% ; 3=50-74 \% ; 4=$ $>75 \%$. This scoring was also performed using the $17 \mathrm{seg}$ ment model. Differentiation between whether patterns of LGE were considered diffuse or confluent was not performed. The mean wall thickness was calculated according to ranked perfusion and LGE scores and tested for significance against zero scores. For perfusion scores $>2$, odds ratios were calculated for increasing degrees of transmurality of LGE and similarly odds ratios were performed for 
LGE scores $>2$ and corresponding perfusion abnormalities.

\section{Results}

The severity of microvascular perfusion abnormalities worsens as wall thickness increases (Figure 1). As wall thickness increases, the percentage transmurality of fibrosis also increases (Figure 2). For those with significant perfusion defects (Perfusion score $>2$ ) the odds ratio of greater degrees of fibrosis also increases (Perfusion score $>$ 2, No LGE OR 1.00, >75\% transmural LGE OR 5.93). Similarly, for those with significant degrees of fibrosis (LGE > 2 ), the as perfusion abnormalities worsen, the odds ratio (OR) of greater degrees of fibrosis also increases (LGE score $>2$; No perfusion abnormality OR 1.00 ; $>75 \%$ perfusion abnormality OR 12.53). While perfusion and LGE areas were closely linked, the transmurality of LGE often exceeded that of the perfusion defect and vice versa $(\mathrm{k}=$ $0.192,95 \%$ CI: $0.148-0.236, \mathrm{p}<0.001$ ).

\section{Conclusion}

This study suggests that areas of maximal hypertrophy in HCM are at greater ischaemic risk and develop greater degrees of fibrosis, possibly due to microvascular ischaemia. Fibrosis and microvascular ischaemia may represent novel risk markers for arrhythmic events and potential therapeutic targets. The close association between degrees of fibrosis and perfusion defect may suggest a causal relationship but the lack of association between absolute transmurality scores between these two variables implies other pathophysiological mechanisms may also contribute.

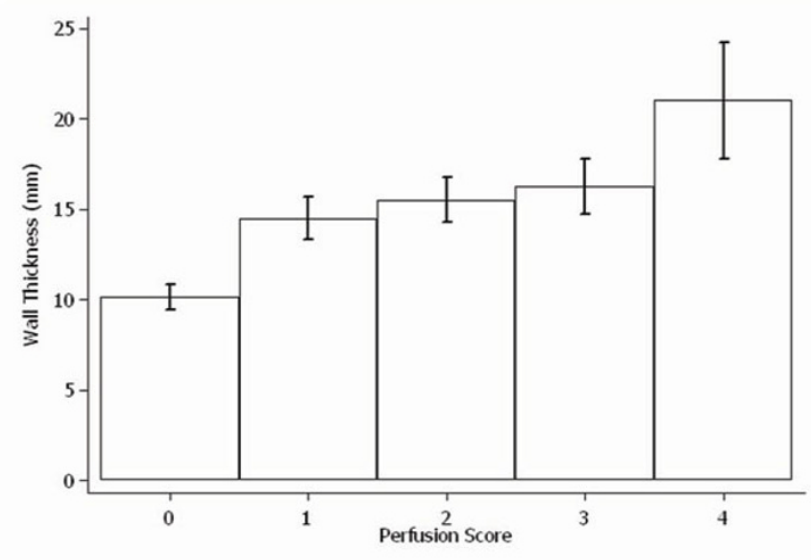

Figure I

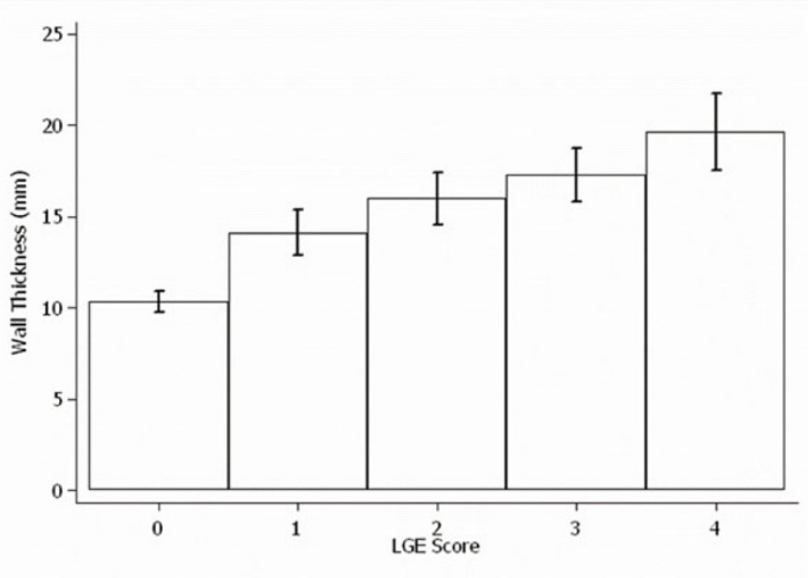

Figure 2 